\title{
Hypericum gentianoides Produces Bioactive Compounds in Schizogenously Formed Glands
}

Heather L Babka*, Matthew L. Hillwig*, Jason Price ${ }^{* *}$, Wendy Maury ${ }^{* *}$, Hilal Ilarslan*, Lankun $\mathrm{Wu}^{*}$, Eve Syrkin Wurtele

* Department of Genetics, Development, and Cell Biology, Iowa State University, Ames, IA 50011

** Department of Microbiology, University of Iowa, Iowa City, Iowa 52242

The genus Hypericum is home to a number of plant species that have promising medicinal properties. Hypericum gentianoides is a North American species formerly utilized medicinally by Cherokee Native Americans [1]. Several unusual acylphloroglucinols that may induce immune responses in mammalian macrophages have recently been discovered in this species [2]. Here, we report several bioactivities of $H$. gentianoides extracts on mammalian cells and describe the ontology of secretory glands accumulating these phytochemicals in planta. Hypericum gentianoides extracts alter calcium homeostasis in HEK293 cells and rat astrocytes. In addition extracts from $H$. gentianoides inhibit the infectivity of human immunodeficiency virus (HIV) on HeLa37 cells in infectivity assays.

Saroaspidin A, uliginosin A, and hyperbrasilol C, the major acyl-phloroglucinols of $H$. gentianoides, accumulate within translucent glands found on most above-ground plant parts. Translucent glands found on $H$. gentianoides develop schizogenously, i.e., secretory cells contained within these glands pull away from one another to create an open cavity (Fig. 1). The secretory cells contained within young translucent glands contain dense cytoplasm packed with a network of ER, ribosomes, and specialized plastids with multidirectional tubuli. Mitochondria begin to visibly degenerate in young glands and have completely disappeared by maturity. As glands age and secretory cells pull apart, bulbous protrusions appear along the cell walls, and material with a fibrillar appearance is present along secretory cell walls (Fig. 2). Vesicle-like structures, stained dark by osmium tetroxide, occur along secretory cells walls, presumably associated with the release of secretory product into the lumen. When glands reach maturity, secretory cell size is reduced as the luminal space becomes filled with secretory product. The intensity of the osmium staining in the peripheral and surrounding cells decreases as glands age, suggestive of concomitant changes in phytochemical contents of these cells (Fig. 2).

\section{References}

[1] Hamel, P. and Chiltoskey, M., Cherokee plants and their uses -- A 400 year history: Sylva: Herald Publishing Co. (1975).

[2] M.L. Hillwig et al., J. Agric. Food Chem., 56 (12) (2008) 4359-4366

Acknowledgements. The microscopy was conducted at the Microscopy and NanoImaging Facility (MNIF) at Iowa State University. 


\section{Translucent Gland Stages}
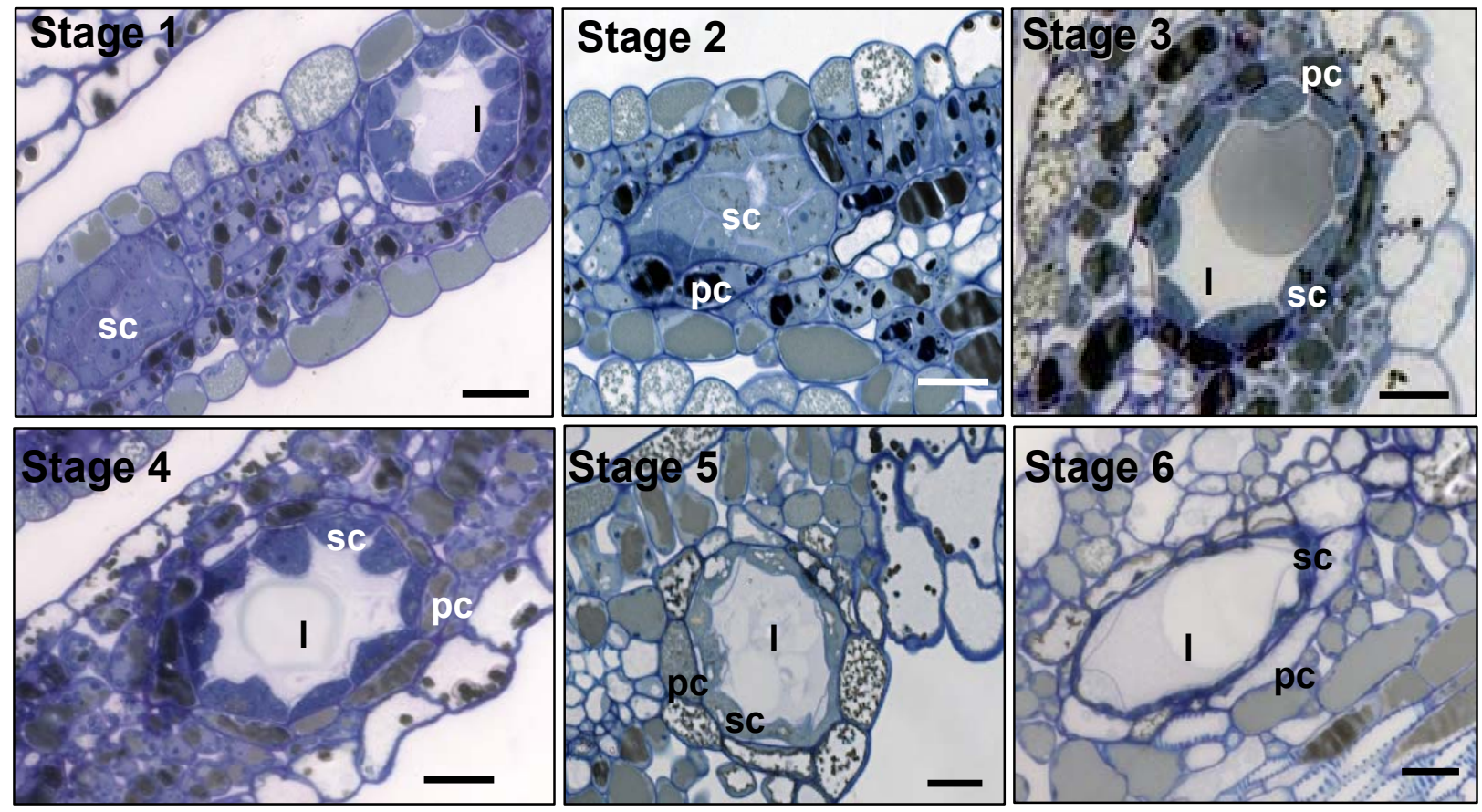

FIG. 1. Light Microcopy of development of Hypericum gentianoides glands. Bars $=20 \mu \mathrm{m}$.
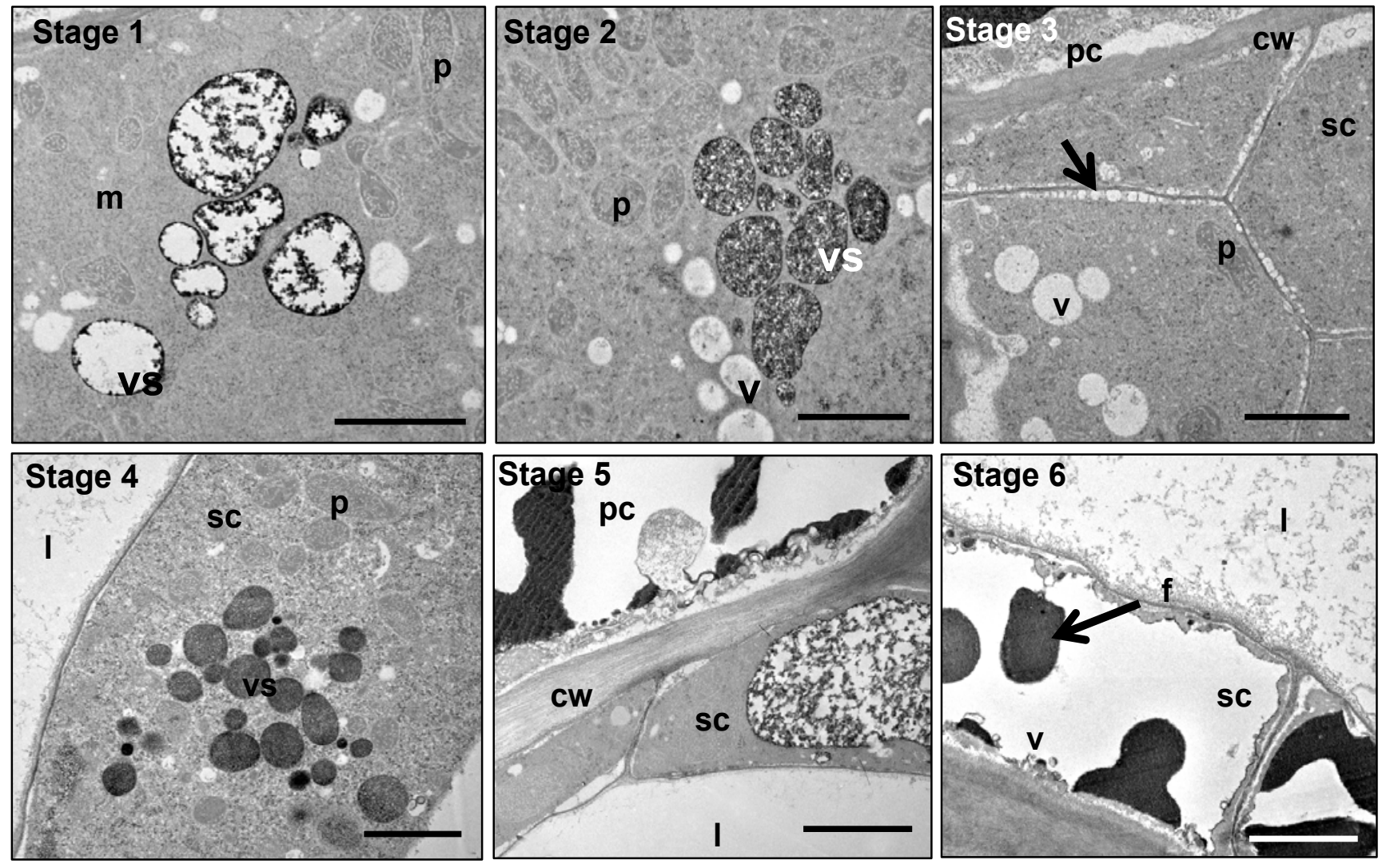

FIG. 2. Transmission Electron Microcopy of development of Hypericum gentianoides glands. pc - peripheral cell; sc - secretory cell; I - lumen, f- fibrillar material; $m$ - mitochondria; $p$ plastid; v - vacuole; vs - vesicle-like body. Bars $=2 \mu \mathrm{m}$. 